\title{
Gluon dominance model
}

\author{
Elena Kokoulina ${ }^{1,2, \star}$ and Andrey Kutov ${ }^{3, \star \star}$ \\ 1 Joint Institute for Nuclear Research, Dubna, Moscow region, 141980 Russia \\ ${ }^{2}$ P.O.Sukhoi Gomel State Technical University, Gomel, 246000 Belarus \\ ${ }^{3}$ Department of Mathematics, Ural Division, RAS, Syktyvkar, 167982 Russia
}

\begin{abstract}
Study of multi-particle production has longer than the semi-centennial history. As it is known, with the growth of energy of accelerators, the new channels of reaction are being opened, the average number of secondary particles is increasing. Physicists are able to accelerate stable particles, such as electrons, positrons, protons, antiprotons, ions (both light and heavy). Rarely, they accelerate kaons and pions. The obtained experimental material stimulates the development of the different theoretical approaches. Since appearance of the modern theory of strong interactions, quantum chromodynamics (QCD), our understanding of multi-particle production is advanced significantly. The language of quarks and gluons is basic one at the explanation of observable phenomena. This review is devoted to the history of appearance and the following development of the gluon dominance model. This model is based on the pQCD and the phenomenological description of the hadronization stage. It permits to describe multiplicity distributions both for lepton and hadron interactions, especially in the high multiplicity region.
\end{abstract}

\section{Introduction}

Study of the multi-particle processes had become very popular in the middle of seventies $[1,2]$. It has been found that in a collision of two relativistic particles $a$ and $b$, three and more hadrons can be produced: $a+b \rightarrow c_{1}+c_{2}+\cdots+c_{n}$. The number of secondary particles, $n$, is called multiplicity. In the experiments, we distinguish multiplicity of charged, $n_{c h}$, and neutral, $n_{0}$, particles. Sum of charged and neutral particles is the total multiplicity: $n_{\mathrm{tot}}=n_{\mathrm{ch}}+n_{0}$.

For the description of multi-particle processes, the language of the mathematical statistics is applied [3]. The multiplicity distribution (MD), $P_{n}$, or the probability of production of $n$ particles is the ratio $P_{n}=\sigma_{n} / \sigma_{\text {inel }}$, where $\sigma_{n}$ is the topological cross section, $\sigma_{\text {inel }}=\sum_{n} \sigma_{n}$. The $k$ th moment of $P_{n}$ is $<n^{k}>=\overline{n^{k}}=\sum_{n} n^{k} P_{n}$. The moment of the first order is a mean multiplicity (an average value), $\bar{n}$. The $k$ th central moment is $\left\langle(n-<n>)^{k}\right\rangle=\overline{(n-\bar{n})^{k}}$. The central moment of the second order is a variance, $D_{2}=\overline{n^{2}}-\bar{n}^{2}=\sigma^{2}$, where the standard deviation $\sigma$ is its square-root.

The number of secondary particles varies from event to event. But on average, the mean multiplicity is staying constant at the given energy and for the given interaction. The events in which the number of secondary particles considerably exceeds mean multiplicity are called the high multiplicity

\footnotetext{
${ }^{\star}$ e-mail: kokoulina@jinr.ru

$\star \star$ e-mail: akutov@rambler.ru
} 
(HM) events and this region - high multiplicity region (HMR). Since last years, the HM event study has drawn a high attention that is connected with discovery of the collective behaviour of secondaries in nuclear and hadron interactions (ridges, flows, shock waves) [4-6].

Our SVD-2 Collaboration studies the HM events in a proton-proton, a proton-nucleus and a nucleus-nucleus interactions at the U-70 accelerator (IHEP, Protvino) and at Nuclotron (JINR, Dubna). Before beginning of these experiments, to predict the topological cross sections at the HMR, the gluon dominance model [7] has been developed. It evolved from the two stage model (TSM) [8] worked out for the description of MD in $e^{+} e^{-}$annihilation at high energies. Both models are based on the convolution of two main stages. In accordance of QCD, on the first stage, a quark-gluon cascade is developed. The second stage is hadronization. For this stage a phenomenological scheme is applied. Comparison of these models with experimental data on MD and other values shows good agreement.

Study of MD is important for understanding of the multi-particle production mechanism. TSM confirms the a fragmentation mechanism of hadronization in $e^{+} e^{-}$annihilation and GDM - a recombination mechanism in hadron interactions. Both models evidence an active role of gluons and a passivity of valency quarks at the multi-particle production.

This paper is organized as follows. In section 2, we describe the construction of TSM and its main results. In section 3, we develop this scheme to study hadron interactions and we call it GDM. It has been shown that the region of HM is stipulated for a gluon fission which can explain the tail of HM and even the ridge phenomenon [9]. The search for collective phenomena in $p p$ interactions in HMR is carried out by the SVD-2 Collaboration at U-70 and Nuclotron. The growth of a scaled variance in HMR of the total multiplicity can be the indication the pion (Bose-Einstein) condensate formation [10]. Then we inform about our searching for the possible connection of the pion condensate and an excess yield of soft photons (whose energy is smaller than $50 \mathrm{MeV}$ ) in AA interactions at Nuclotron [11]. We round off with our future plans and conclusions in section 4.

\section{TSM and $e^{+} e^{-}$annihilation}

TSM has been constructed in the end of seventies [14]. It was aimed at the MD description of hadrons in the $e^{+} e^{-}$annihilation at high energies. In accordance with $\mathrm{QCD}$, this process is realized through $\gamma$ or $Z^{0}$-boson with formation of a quark-antiquark pair, its branching and a conversion of the initial and the newly born quarks and gluons to the observable hadrons: $e^{+} e^{-} \rightarrow q \bar{q} \rightarrow(q, \bar{q}, g) \rightarrow h_{1}+h_{2}+\cdots+h_{n}$. If quarks have sufficiently high energy, they can develop quark-gluon cascade $(q, \bar{q}, g)$. In $[12,13]$ three elementary processes are used in this description: 1) $q \rightarrow q+g$,2) $g \rightarrow g+g$ and 3) $g \rightarrow q+\bar{q}$. The first two from them are predominated over the third one until the cascade is developed. Giovannini [12] has constructed and decided the system of the differential equations for the Markov branching process of quark and gluon. He writes them on the language of the generating functions (GF), $Q(z)=\sum_{n=0}^{\infty} P_{n} z^{n}$, where $z$ - the auxiliary parameter. GF $Q(z)$ is connected with MD $P_{n}$ :

$$
P_{n}=\left.\frac{1}{n !} \frac{\partial^{n}}{\partial z^{n}} Q(z)\right|_{z=0}
$$

The $k$ th moment of MD is also calculated through the differentiation of GF: $\overline{n(n-1) \ldots(n-k+1)}=$ $\partial^{n} Q(z) /\left.\partial z^{n}\right|_{z=1}$. For the quark jet, the $q g$-cascade leads to the negative binomial distribution (NBD):

$$
P_{m}^{q}=\frac{k_{p}\left(k_{p}+1\right) \ldots\left(k_{p}+m-1\right.}{m !}\left(\frac{\bar{m}}{\bar{m}+k_{p}}\right)^{m}\left(\frac{k_{p}}{\bar{m}+k_{p}}\right)^{k_{p}}
$$

with GF

$$
Q^{q}(z)=\left[1+\frac{\bar{m}}{k_{p}}(1-z)\right]^{-k_{p}}
$$


where $k_{p}$ is the ratio of probabilities of two elementary processes 1) and 2): a quark bremsstrahlung of a gluon to a gluon fission, $\bar{m}-$ a mean multiplicity of gluons.

$\mathrm{MD}$ for a $g$-jet is the Farry distribution with GF $Q^{g}(z)$

$$
P_{m}^{g}=\frac{1}{\bar{m}}\left(1-\frac{1}{\bar{m}}\right)^{m-1}, \quad Q^{g}(z)=\frac{z}{\bar{m}}\left[1-z\left(1-\frac{1}{\bar{m}}\right)\right]^{-1}
$$

To get MD of hadrons, it is necessary to take into account a hadronization. From experimental data, it is known that the second correlative moment, $f_{2}=\overline{n(n-1)}-\bar{n}^{2}$, for $e^{+} e^{-}$annihilation is negative at low energy. With growth of energy, it changes sign from "minus" to "plus" [15]. That behaviour is typical for a multi-particle production. Obviously, at the low energy, the $q g$-cascade is not enough developed. In this case the hadronization carries in MD the determinative contribution. As MD at hadronization stage has the negative second correlative moment, a binomial distribution (BD) has been chosen for this stage

$$
P_{n}^{h}=\left(\begin{array}{c}
N_{P} \\
n
\end{array}\right)\left(\frac{\bar{n}_{P}^{h}}{N_{P}}\right)^{n}\left(1-\frac{\bar{n}_{P}^{h}}{N_{P}}\right)^{N_{P}-n}
$$

where $\bar{n}_{P}^{h}$ and $N_{P}$ are parameters having the sense of the mean multiplicity and maximum number of hadrons formed from a single parton $P=q, g$ (quark or gluon) through its passing of the hadronization stage. The second correlative moment for BD is equal to $f_{2}=-\left(\bar{n}_{P}^{h}\right)^{2} / N_{P}<0$. In the case of independent particle production which is described by Poisson distribution, $P_{n}=e^{-\bar{n}} \bar{n}^{n} / n$ ! with GF $Q(z)=e^{\bar{n}(z-1)}, f_{2} \equiv 0$. NBD gives positive $f_{2}=\bar{m}^{2} / k_{p}$.

The convolution of this two stages is based on the hypothesis of the soft discolouration [14] and an equality of the hadron production probabilities from quark and gluon at the second stage. It leads to final expression for MD of hadrons in $e^{+} e^{-}$annihilation [8]

$$
P_{n}=\Omega \sum_{m=0}^{M_{g}} P_{m}^{q}\left(\begin{array}{c}
(2+\alpha m) N \\
n
\end{array}\right)\left(\frac{\bar{n}^{h}}{N}\right)^{n}\left(1-\frac{\bar{n}^{h}}{N}\right)^{(2+\alpha m) N-n}
$$

where $\Omega$ is a normalization factor, $N=N_{q}, \alpha=N_{g} / N_{q}, M_{g}$ is the maximum number of active gluons (they can fragment to hadrons). TSM gives good description of MD in $e^{+} e^{-}$annihilation from 9 up to $189 \mathrm{GeV}[8,16]$. In figure 1 , on the left panel, the description of MD at $160 \mathrm{GeV}$ [17] by TSM is shown. The mean multiplicity of gluons after the completion of the $q g$-cascade is described well by the logarithmic dependence with energy. The parameter of hadronization for a quark, $\bar{n}_{q}^{h}$ varies in the wide limits. In contrast to the quark parameter, the gluon parameter, $\bar{n}_{g}^{h}$ has a value close to one, that is confirmed by fitting: $\bar{n}_{g}^{h}=0.947 \pm 0.002$ at $\chi^{2} / \mathrm{ndf}=34 / 17=2$ (figure 1 , the right panel). The TSM confirms predicted by I. Dremin [3] oscillations of the ratio of cumulative factorial moment to factorial moment as function of their rank, the growth of the period of oscillations at energy higher 90 $\mathrm{GeV}$ and changing of the sign of the second correlative moment with energy $[8,14]$

$$
f_{2}=\left[\alpha^{2} \frac{\bar{m}^{2}}{k_{p}}+\alpha^{2} \bar{m}-\frac{2+\alpha \bar{m}}{N}\right]\left(\bar{n}^{h}\right)^{2} .
$$

The characteristics of the gluon jet obtained with TSM have been used in the description of MD in the tree-gluon decay of quarkonia, $\Upsilon(9.46)$ and $\Upsilon$ (10.02) [18]

$$
P_{n}\left(m_{\Upsilon}\right)=\sum_{m^{\prime}=0} \frac{\left(m^{\prime}-1\right)\left(m^{\prime}-2\right)}{2(\bar{m} / 3)^{3}}\left(1-\frac{1}{\bar{m} / 3}\right)^{m^{\prime}}\left(\begin{array}{c}
\left(3+m^{\prime}\right) N_{g} \\
n
\end{array}\right)\left(\frac{\bar{n}_{g}^{h}}{N_{g}}\right)^{n}\left(1-\frac{\bar{n}_{g}^{h}}{N_{g}}\right)^{\left(3+m^{\prime}\right) N_{g}-n}
$$



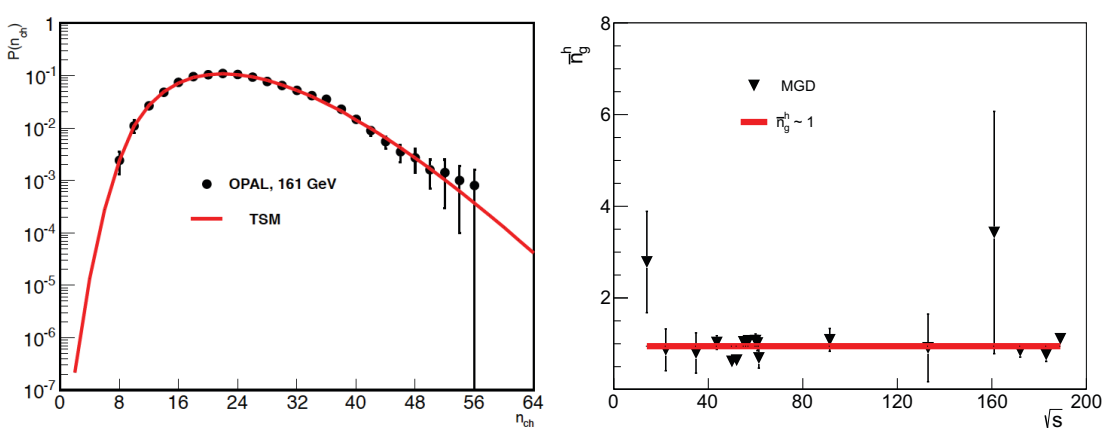

Figure 1. Left panel: The description of MD by TSM in $e^{+} e^{-}$annihilation at $161 \mathrm{GeV}$ [17]. Right panel: the gluon parameter of hadronization versus energy.

The differences between mean multiplicity of hadrons in the region with two jet formation close to quarkonium and multiplicity of the three-gluon decay calculated in TSM is identical to the experimental value.

Using TSM we estimate values of mean multiplicity for $e^{+} e^{-}$annihilation at the energies of future ILC and CLIC accelerators: $\bar{n}(500 \mathrm{GeV}) \approx 30 \pm 5, \bar{n}(1 \mathrm{TeV}) \approx 50 \pm 10$.

\section{GDM and hadron interactions}

The "Thermalization" project was aimed at the collective phenomena searching for in HM region in $p p$ interactions at the U-70 accelerator in IHEP (Protvino). The energy of a proton beam was $50 \mathrm{GeV}$. We have manufactured a HM-trigger to suppress registration of events with a small multiplicity. This allowed us to go down on the topological cross sections by three orders reaching max $n_{c h}=24$ (at 50 $\mathrm{GeV}$ the kinematical limit is equal to 59 pions). At this energy, $\mathrm{HM} n>>\bar{n} \approx 5$ for charged particles.

Before beginning of the "Thermalization" project, our SVD-2 Collaboration has found out that the Monte Carlo simulation underestimates the experimental data of the Mirabelle Collaboration [19] for topological cross section at the maximum value of the measured multiplicity, $n_{c h}=18$, two orders in $p p$ interactions at $70 \mathrm{GeV}$ proton beam [20]. The phenomenological models gave them the different predictions too. One of them is MGD [7], called a gluon model, second one - quark model [21].

To modify TSM for hadron interactions, we have supposed that in hadron interactions, a valence quarks and the nascent gluons develop branching in accordance to QCD elementary processes.

The valency quark inclusion led us to values of $\bar{h}^{h}$ « 1 . With decrease of the number of valence quarks, this parameter begins to grow. Only completely an excluding of the valence quark (they are staying in the leading particles), we achieve the considerable improvement of $\chi^{2}$, at that, the hadronization parameters become rather more than 1 as in the $e^{+} e^{-}$annihilation. It indicates the change of mechanism of hadronization: from fragmentation to recombination one [7].

This result has permitted us to formulate a gluon dominance model (GDM). According to this model, the basic sources of secondary hadrons are gluons, we call them active ones. As in TSM, the first stage is a formation of $q g$-medium and its branching, the second stage is hadronization of gluons. GDM exists in two schemes, with a fission of gluon and without of it. The first scheme testifies to half of the nascent gluons fragment in hadrons. The rest of them are caching up by quarks and reradiating can be sources of the soft photons (SP). MD of hadron in the second scheme presents the convolution 

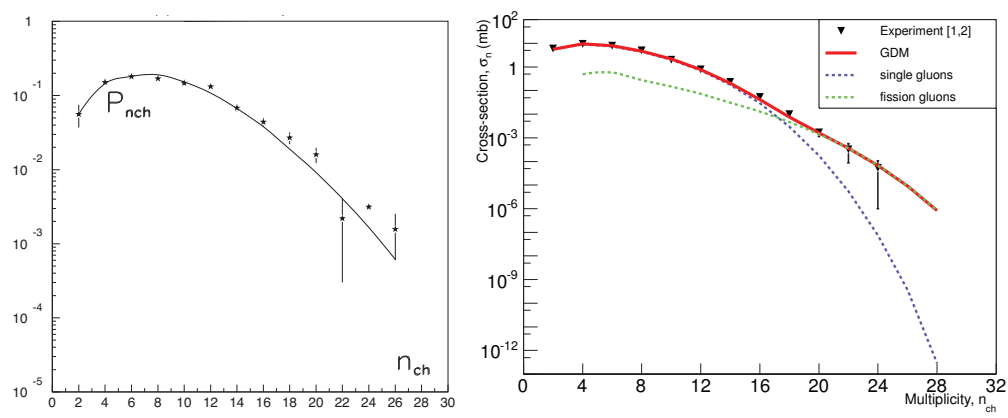

Figure 2. The description of MD by GDM in $p p$ interactions. Left panel: at $300 \mathrm{GeV} / c$ [22]; Right panel: at 50 $\mathrm{GeV} / c$ with taking into account the gluonic fission [23].

of two stages in the case of the independent appearance of active gluons

$$
P_{n}=\sum_{m=1}^{M} \frac{\bar{m}^{m} e^{-\bar{m}}}{m !}\left(\begin{array}{c}
m N \\
n-2
\end{array}\right)\left(\frac{\bar{n}^{h}}{N}\right)^{n-2}\left(1-\frac{\bar{n}^{h}}{N}\right)^{m N-(n-2)} .
$$

In figure 2, left panel the description of MD at $300 \mathrm{GeV}$ [22] by GDM is offered. In [9] it has been shown that with increasing of energy, a gluon fission becomes predominant in comparison to quark bremsstrahlung. In figure 2, right panel, the convolution of two contributions (without and with gluon splitting) describes well MD, including HM region.

The differences of topological cross sections $p \bar{p}$ annihilation and $p p$ collisions at the same energy reveal two local maximums [24]. This two-humped structure can be described in the framework of GDM. For this purpose, some intermediate charged topologies determined by the valency quark combination have been introduced [25]. In the HM region, the "4"-topology (participate valency and sea quarks) carries noticeable contribution in these differences in HMR.

\section{Pionic condensate and soft photons. Conclusion.}

The SVD-2 Collaboration has received indication at the formation of the Bose-Einstein (pionic) condensate (BEC) in the high total multiplicity [26]. Begun and Gorenstein [27] within the framework of the ideal hadron gas model have shown that the sharp growth of the scaled variance, $\omega^{0}$ (the ratio of a variance to mean multiplicity of the neutral pions at a given total multiplicity) is the signal of BEC formation. Our experiment testifies that the region of high total multiplicity is a very interesting one. The two-particle Bose-Einstein correlations in $p p$ collisions at $\sqrt{s}=0.9$ and $7 \mathrm{TeV}$ measured with the ATLAS detector in the HM region show that the behaviour of their parameters testifies about approaching to the BEC ( $\lambda \rightarrow 0$ - fully coherent emission of identical bosons, the effective radius $R$ is staying constant or its measurement is difficult) [28].

Registration and research of the SP yield in hadron and nuclear interaction were the tasks standing before our experiment. There are some theoretical models which explain the excess of their yield by the BEC formation. That is why we have designed and manufactured an electromagnetic calorimeter with the low energy threshold smaller than $50 \mathrm{GeV}$ [29]. Our first measurements confirmed the excess yield of SP in interactions of deuterium and lithium beams with a carbon target at $5 \mathrm{~A} \mathrm{GeV} / c$ 
at Nuclotron. In the nearest future we plan studying: a connection between BEC formation and an excess of SP yield; a search of new resonances in the system of two $\gamma$-quanta $\left(f_{0}(500)\right.$ or $\sigma$-meson, etc.); a study of increased yield of $\eta^{0}$-mesons in AA-interactions; research of pair photon interference ( $\gamma$-femtometry); a search of P-parity violation effect in the events with high $p_{T}$ in the BM@ $\mathrm{N}$ setup.

\section{References}

[1] R. Hagedorn, Nuovo Cim. Suppl. 3, 147-186 (1965)

[2] V.S. Murzin and L.I. Sarycheva, Physics of hadron processes (Energoatomizdat, Moscow, 1986, in Rissian) $280 \mathrm{p}$.

[3] I.M. Dremin, Phys. Lett. B 341, 95-98 (1994)

[4] J.Adams et al. STAR Collaboration, Nucl. Phys. A 757, 102-183 (2005)

[5] K.Adcox et al. PHENIX Collaboration, Nucl. Phys. A 757, 184-283 (2005)

[6] S.Chatrchyan et al. JHEP 9, 111-132 (2012)

[7] E. Kokoulina, A. Kutov, and V. Nikitin, Braz. J. Phys. 37, 2C, 785-787 (2007)

[8] E.C. Kokoulina, in the proc. of XXXII ISMD, Alushta, Ukraine (World Scientific, 2002) 340

[9] E.A. Kuraev, E. A. S. Bakmaev and E.S. Kokoulina, Nucl. Phys. B 851, 551-564 (2011)

[10] A.G. Afonin et al. SVD-2 Collaboration, EPJ Web of Conferences 37, 06002 (2013)

[11] E. Ardashev et al. Eur.Phys.J. A 52, 261 (2016)

[12] A. Giovannini, Nucl. Phys. B 161, 429-448 (1979)

[13] K. Konishi, A. Ukawa and G. Veneziano, Nucl. Phys. B 157, 45-107 (1979)

[14] V.I. Kuvshinov and E.S. Kokoulina, Acta Phys.Pol. B 13, 533 (1982)

[15] J.G. Rushbrooke and B.R. Webber, Phys. Rep. 44, 1-92 (1978)

[16] E.C. Kokoulina, In proceedings of11th Annual Seminar on Nonlinear Phenomena in Complex Systems: Chaos, Fractals, Phase Transitions, Selforganization. Minsk, Belarus, 13-16 May 2002, 11, 23-136 (2003)

[17] K.Ackerstaff et al., The OPAL Collaboration, Z.Physics C 75, 193-207 (1997)

[18] E.S. Kokoulina and V.I. Kuvshinov, Sov.Phys.J. 28, 757-760 (1985)

[19] V.V.Ammosov et al., Phys. Let. B 42, 519-521 (1972)

[20] E. Kokoulina, Acta Phys. Polon. 35, 295-302 (2004)

[21] O.G. Chikilev and V.P. Chlyapnikov, Phys. Atom. Nucl. 55, 779-783 (1992)

[22] A. Firestone et al., Phys. Rev. D 10, 2080-2083 (1974)

[23] E.S. Kokoulina, Phys.Part.Nucl.Lett. 13(1), 74-78 (2016)

[24] J.G. Rushbrooke and B.R. Webber, Phys. Rep. 44, 1-92 (1978)

[25] E.C. Kokoulina, American Institute of Physics. Conference proceedings 828, 81-86 (2006)

[26] E. Kokoulina, Progr. of Theor. Phys. Suppl. 193, 306-309 (2012)

[27] V.V. Begun and M.I. Gorenstein, Phys. Rev. C 77, 064903 (2008)

[28] ATLAS Collaboration, Eur. Phys. J. C 75, 466 (2015)

[29] E. Ardashev et al., Eur. Phys. J. A 52(8), 261 (2016) 